TI 2015-065/VIII

Tinbergen Institute Discussion Paper

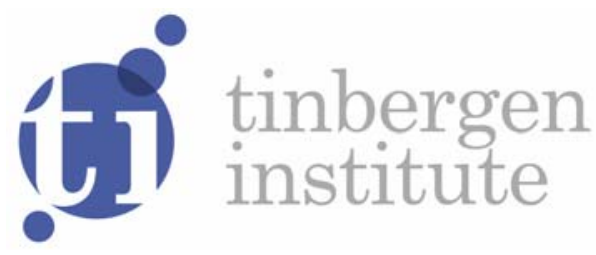

\title{
A Theory of Continuous Uncertainty Types
}

\author{
Achim I. Czerny 1,2 \\ Erik T. Verhoefl,2 \\ Anming Zhang 3
}

${ }^{\prime}$ Faculty of Economics and Business Administration, VU University Amsterdam, the Netherlands;

2 Tinbergen Institute, the Netherlands;

3 University of British Columbia, Canada. 
Tinbergen Institute is the graduate school and research institute in economics of Erasmus University Rotterdam, the University of Amsterdam and VU University Amsterdam.

More TI discussion papers can be downloaded at http://www.tinbergen.nl

Tinbergen Institute has two locations:

Tinbergen Institute Amsterdam

Gustav Mahlerplein 117

1082 MS Amsterdam

The Netherlands

Tel.: +31(0)205251600

Tinbergen Institute Rotterdam

Burg. Oudlaan 50

3062 PA Rotterdam

The Netherlands

Tel.: +31(0)10 4088900

Fax: $+31(0) 104089031$

Duisenberg school of finance is a collaboration of the Dutch financial sector and universities, with the ambition to support innovative research and offer top quality academic education in core areas of finance.

DSF research papers can be downloaded at: http://www.dsf.nl/

Duisenberg school of finance

Gustav Mahlerplein 117

1082 MS Amsterdam

The Netherlands

Tel.: +31(0)20 5258579 


\title{
A Theory of Continuous Uncertainty Types ${ }^{1}$
}

\author{
Achim I. Czerny ${ }^{2}$, Erik T. Verhoef ${ }^{3}$ and Anming Zhang ${ }^{4}$
}

May 28, 2015

${ }^{1}$ We thank Alex Anas, Jan Brueckner, Mogens Fosgerau, Marc Ivaldi, Sergio Jara-Diaz, Paul Koster, Jos van Ommeren, Vincent van den Berg, Jaques Thisse and the participants of the EUREKA seminar at the VU University Amsterdam and the OPTION Conference for helpful comments and suggestions. Partial financial support from the European Research Council (ERC, AdG Grant $\sharp 246969$ OPTION) is gratefully acknowledged.

${ }^{2}$ Department of Spatial Economics, VU University Amsterdam, and Tinbergen Institute, The Netherlands, a.i.czerny@vu.nl.

${ }^{3}$ Department of Spatial Economics, VU University Amsterdam, and Tinbergen Institute, The Netherlands, e.t.verhoef@vu.nl.

${ }^{4}$ Sauder School of Business, University of British Columbia, Canada, anming.zhang@sauder.ubc.ca. 


\begin{abstract}
This paper distinguishes uncertainty types that differ continuously with respect to the degree to which uncertainty affects the optimal price/price markup or optimal quantity. A monopoly example is used to show that seemingly strong assumptions on functional forms can represent a wide variety of different scenarios, while (implicit) assumptions on continuous uncertainty types can lead to quite special results. Monopoly examples of the newsboy problem type are further used to show that the optimal capacity level and the optimal composition of capacity in terms of the number and size of production units depends crucially on the type of uncertainty and the employed functional forms for utilities and costs.
\end{abstract}

Keywords: Continuous uncertainty types; demand uncertainty; cost uncertainty; monopoly; newsboy problem.

JEL: D42; D80; L91. 


\section{Introduction}

Capacity decisions are typically long term relative to pricing decisions: Firms can relatively easily adjust prices to volatile market fluctuations; but, capacity often limits output in periods of high demand, whilst excess capacity exists in periods of low demand, which usually cannot be easily fixed in the short run. ${ }^{1}$ On top of this, periods of high and low demand may be difficult to predict, meaning that capacity decisions have to be made under uncertainty. Airlines, rail operating companies, cruise and passenger lines, container lines, freight forwarders and logistics companies can serve as examples. These firms need to plan their fleet of vehicles long term. Furthermore, they typically face significant fluctuations in demand and cost conditions, some of which may be caused by predictable seasonal patterns but others are hard to foresee at the time when capacity decisions are made. Both types of fluctuation, whether predictable or not, pose challenges for capacity choice, however. Also in infrastructure supply (airports, seaports, highways, rail tracks, etc.) and in other businesses and institutions such as hotels, cinemas, schools, hospitals and fire fighters, where capacity may be determined by the size of facilities or the number of workers, similar issues emerge.

A general question is how uncertain fluctuations in demand and costs possibly affect firm behavior in terms of price and capacity. Some insights can be found in the literature, which typically distinguishes between two (i.e., discrete) types of uncertainties called "additive" and "multiplicative" uncertainties. For example, let $D$ denote the deterministic part of the demand and $\mu$ be a noise terms, then additive uncertainty means that the noise term is added to the deterministic part of the demand, i.e., demand is $D+\mu$, while multiplicative uncertainty means that the demand is determined by product of the deterministic and the stochastic parts of the demand, i.e., demand is $\mu D$. In this example, the additive uncertainty can be interpreted as a situation where the effect of uncertainty (measured by the standard deviation of the noise term) on the willingness to pay is the same for all customers and, thus, independent of the level of willingness to pay. On the other hand, with

\footnotetext{
${ }^{1}$ Meyer (1975) shows that it can indeed be optimal for a monopoly firm to invest in production capacity that exceeds production in the case of low demand.
} 
multiplicative uncertainty the willingness to pay of customers with a high willingness to pay may be increasing in the noise term, while the willingness to pay of customers with a low willingness to pay may be decreasing in uncertainty.

This paper is closely related to the large body of literature on the so called newsboy problem. More specifically, the newsboy problem describes a periodicreview inventory model, where a firm (typically a monopoly) decides its inventory facing uncertain demand for perishable products (e.g., Massé, 1946; Arrow et al., 1951; Dvoretzky et al., 1952a and 1952b; Within, 1955). For example, Mills (1959) considered a monopolistic firm and additive demand uncertainty, and found that uncertainty can reduce the ex-ante optimal price because the firm this way reduces the probability of unsold production. Karlin and Carr (1962) found that with multiplicative uncertainty the opposite is true, i.e., uncertainty increases the ex-ante optimal price. Also Zabel (1972) found that monopoly behavior depends on the structure of stochastic processes: with additive uncertainty, output increases in uncertainty (measured by the standard deviation of the noise term), while output decreases in uncertainty with multiplicative uncertainty. For public utility pricing, Brown and Johnson (1969) found that optimized prices can be decreasing in uncertainty and optimized output increasing in uncertainty independent of whether demand uncertainty is additive or multiplicative. Furthermore, for passenger management in the airline industry, newsboy model has been used for single-leg revenue management problems (McGill and van Ryzin, 1999). Wong et al. (2009) consider passengers and cargo and is a newsvendor model of multiple items with capacity constraints. They also address multiple fare classes and price-dependent demand. This means that it is a newsboy model that incorporates the relationship between pricing and stock quantity (Polatoglu, 1991; Gallego and Ryzin, 1994; Weatherford, 1997; Petruzzi and Dada, 1999; Khouja, 2000).

In this paper we aim to contribute to the literature by developing a general theory of continuous uncertainty types. The theory is general in the sense that it can be used to provide a comparative statics analysis of economic results, e.g., monopoly behavior or the behavior of a social maximizer, in uncertainty types. This feature is useful because it can be used to derive new insights on how the structure of stochastic 
processes can affect economic outcomes. The theory is developed for a risk-neutral monopoly firm that optimizes prices ex post (i.e., after uncertainty has resolved), while capacity decisions on the upper limit on output are optimized ex ante so as to maximize expected profit. $^{2}$ Note that the monopoly problem that we use to develop our exposition is mainly used for illustration; our theory of continuous uncertainty types is not restricted to the monopoly example. ${ }^{3}$ More specifically, our paper proposes to differentiate uncertainty with respect to the degree to which uncertainty affects the optimized price (or price-markup) and the optimized quantity. Then, the level of uncertainty can be measured by the standard deviation of the total effect of uncertainty on the differences of the optimized prices and optimized outputs, relative to the corresponding expected values of these optimized prices and optimized outputs.

We derive two main results on the effect of uncertainty on expected monopoly profit when: (i) there are no capacity constraints, and (ii) uncertainty types (i.e, the degree to which uncertainty affects the optimized price and the optimized quantity) is independent of the specific realizations of noise terms. Another way of describing property (ii) is that the ratio of the differences between the actual and expected values for optimized markups and quantities can be described by a (deterministic) constant value. The results can be described as follows: First, an increase in uncertainty increases the (unconstrained) expected profit only if a higher than expected profit margin is associated with a higher than expected quantitiy, while an increase in uncertainty reduces expected profit otherwise (i.e., when a higher than the expected profit margin is associated with a lower than expected quantity or vice-versa). This shows that assumptions on the form of stochastic processes can be quite decisive for the results. Second, we show that the same uncertainty type can emerge from different commonly used functional forms for utilities and cost. In this sense, assumptions on functional forms for utilities and cost, as they are common in

\footnotetext{
${ }^{2}$ Baron (1970) and Sandmo (1971) showed that production output can be reduced by risk aversion.

${ }^{3}$ To show that our theory of uncertainty types is not tied to the monopoly problem and thus can be considered as a more general theory, it is applied to the policy problem of externality internalization in Appendix C.
} 
the literature, can sometimes be made without loss of generality.

To further illustrate the relevance of the theory of continuous uncertainty types developed in this paper, we analyze the role of uncertainty types on monopoly capacity decisions, where we distinguish between one- and two-dimensional capacity (i.e., single valued in output space, or decomposed in the number and size of production units). Here, we show that a change in the uncertainty type can have a positive or a negative effect on the optimal overall level of capacity and, in addition, affect the optimal composition of capacity in terms of the number and size of units. The main fact underlying these results is that a potentially high optimized quantity is constrained by capacity and therefore does not become effective, which affects the incentives to invest into capacity expansions depending on the assumed functional forms for utlities and costs.

The present paper is also related to studies on multi-period capital investments under uncertainty. Hartman (1972) considers the firms' expenditures in capital and labour, where prices are stochastic in each period and exogenous. He finds that capital investments increase in uncertainty. Pindyck (1982) shows, on the other hand, that the effect of uncertainty on capital investments depend on the skewness of the function that determines the adjustment costs incurred by changes in capacity (the skewness determines the curvature of the marginal adjustment cost function). ${ }^{4}$ If the skew is positive, then high uncertainty leads to more investments, while high uncertainty reduces capital investments if the skew is negative. In Pindyck's scenario, the current price is known to the firms, while the future evolution of prices is stochastic. Pindyck conjectures that the difference in the results to Hartman depend on the difference in stochastic specifications. Additionally, Abel (1983) finds that the skewness of the capacity adjustment cost function affects the relation between the expected growth rates of investments and marginal valuation of capital.

Finally, the present study is connected to the strand of the literature that is concerned with the supply of congested infrastructure under uncertainty. Kraus (1982) and D'Ouville and McDonald (1990) found that optimal road investments

\footnotetext{
${ }^{4}$ Here, the skewness determines whether the marginal adjustment cost function is a convex or concave function.
} 
are increasing in demand uncertainty when there is an ex-ante optimal congestion toll. ${ }^{5}$ This result has been confirmed by the analysis of Arnott, de Palma and Lindsey (1996) who consider an iso-elastic demand function with deterministic demand elasticity and a stochastic shift parameter (i.e., multiplicative uncertainty), in combination with a stochastic road capacity (but no congestion toll). The result has further been confirmed by Proost and van der Loo (2010) for the case of ex-post optimal tolls and stochastic iso-elastic demands (multiplicative demand uncertainty), and by Xiao, Fu and Zhang (2013) for the case of ex-post optimal tolls and linear demands (additive demand uncertainty).

The paper is organized as follows. Section 2 develops a classification of continuous uncertainty types and analyzes the effect of continuous uncertainty types on expected profit when there are no capacity limits. Section 3 identifies functional forms for utilities and costs and stochastic or deterministic parameters that lead to a specific uncertainty type. These functional forms are further used to analyze the role of functional forms for capacity decisions in one and two dimensions in Section 4. Section 5 provides conclusions and discusses avenues for future research.

\section{Continuous Uncertainty types}

\subsection{The basic model}

Consider a risk-neutral monopoly firm that charges a per-unit price $p$ to a representative individual who consumes $q$ units of its good. ${ }^{6}$ The strictly concave utility of the representative consumer is denoted as $B$ with $B=B(q)$ and is stochastic such that $B=B(q ; \alpha, \mu)$, where $\alpha$ is a constant and is called the type-parameter (the exact meaning of the type parameter will be developed in detail below) and $\mu$ is a noise term. The time structure is such that the consumer has perfect knowledge

\footnotetext{
${ }^{5}$ D'Ouville and McDonald (1990) discuss their choice of stochastic processes, which can have the interpretation of an additive uncertainty, in their Footnote 1.

${ }^{6}$ Since the monopoly firm is assumed to be risk neutral, there is no qualitative difference in our setting between a situation where demand and cost conditions are fluctuatings with certainty or uncertainty.
} 
about the realization of noise term $\mu$ when she decides upon consumption. The demand function, denoted $D$ with $D=D(p)$, is implicitly determined by the equilibrium condition $B^{\prime}(q)=p$, and is downward sloping with $D^{\prime}(p)=1 / B^{\prime \prime}\left(B^{\prime \prime}\right.$ is the slope of the inverse demand function) by the concavity of the utility function. The firm's average variable costs (henceforth called "unit costs") are stochastic and denoted $c$ with $c=c(q ; \alpha, \nu)$, where $\nu$ is a noise term and $c^{\prime \prime} \geq 0$, which means that the unit cost is convex in $q$. This leads to surplus $(p-c) \cdot D$, with $p-c$ being the markup. Let $\omega(h(x))$ denote the elasticity of the slope of an arbitrary function $h(x)$ with respect to $x$ with $\omega(h(x))=x h^{\prime \prime} / h^{\prime}$ for $h^{\prime}>0$. This elasticity can be used as a measure for the convexity of function $h .^{7}$ For example, if $\omega(h(x))>0$, function $h$ is strictly convex in $x$, while it is strictly concave if $\omega(h(x))<0$ and linear if $\omega(h(x))=0$. Then, if also the firm has perfect knowledge about the value of the noise terms and the benefit function is sufficiently concave in the sense that $\omega\left(B^{\prime}(q)\right)<-1 / B^{\prime \prime}$, the demand function $D(p)$ is log-concave, which means that the optimized markup, denoted $p^{*}-c^{*}$ (asterisks are used to indicate monopoly solutions), and the optimized quantity, $q^{*}$, are implicitly determined by the first-order condition $D+\left(p-c-c^{\prime} D\right) D^{\prime}=0 .{ }^{8}$

\subsection{The main idea}

We distinguish between different types of uncertainty as follows. Note that uncertainty affects the monopoly behavior only if the segments of the demand and cost functions that determine the optimized markup and the optimized quantity are uncertain. This is an obvious but noteworthy insight, since this immediately shows that there are two broad types of uncertainty: one that has some effect on the optimal quantities and another that has no effect. Clearly, we are interested in the first type, where the relevant part of the demand and cost functions are uncertain. In this situation, uncertainty can affect the optimized profit via two channels: (i) by

\footnotetext{
${ }^{7}$ Aguirre et al. (2010) use a similar convexity measure for demand functions.

${ }^{8}$ Log-concavity of demand is a standard assumption, which ensures that the second-order condition for a profit maximum is satisfied. For a detailed discussion of the log-concavity property, see Bagnoli and Bergstrom (2005).
} 


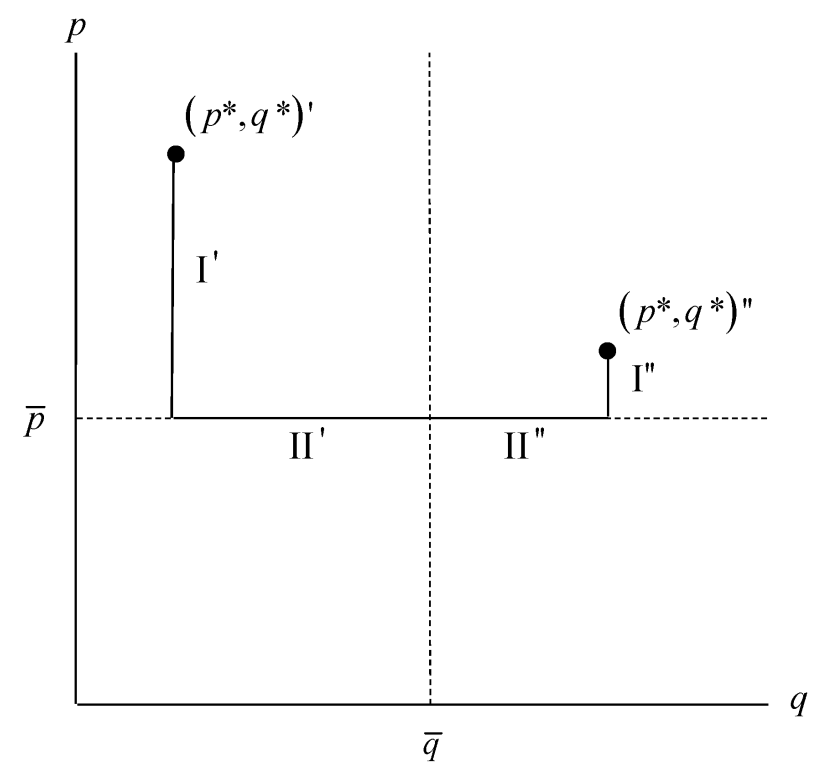

Figure 1: Optimized prices and quantities

changing the optimized markup, and (ii) by changing the optimized quantity. This is the main consideration underlying our categorization of uncertainty types.

Figure 1 illustrates this case for deterministic costs normalized to zero (i.e., $c=0$ ), where the markup is the same as the price. The figure displays the expected optimized price, denoted $\bar{p}$, and the expected optimized quantity, denoted $\bar{q}$, with $\bar{p}<$ $\bar{q}$ and two (of many more) possible realizations of optimized prices and quantities: $\left(p^{*}, q^{*}\right)^{\prime}$ and $\left(p^{*}, q^{*}\right)^{\prime \prime}$ with $\bar{p}<\left(p^{*}\right)^{\prime \prime}<\left(p^{*}\right)^{\prime}$ and $\left(q^{*}\right)^{\prime \prime}>\bar{q}>\left(q^{*}\right)^{\prime}$, i.e., in both states of the demand the optimized prices are higher than expected, while the optimized quantity is higher than expected in one state, $(\cdot)^{\prime \prime}$, and lower than expected in the other state, $(\cdot)^{\prime}$. Note that these two states have the same expected values for optimized prices and quantities only for expositional purposes; but, as described below in detail, this does not mean that they stem from the same population in the sense that the underlying types of uncertainty are different for states $(\cdot)^{\prime \prime}$ and $(\cdot)^{\prime}$. Furthermore, it should be emphasized that $\left(p^{*}, q^{*}\right)^{\prime}$ and $\left(p^{*}, q^{*}\right)^{\prime \prime}$ are both to be interpreted as the mutually consistent values of $p$ and $q$ that the monopolist chooses after becoming aware of the actual realizations of the relevant marginal revenue and marginal cost functions.

To capture the effect of uncertainty on the optimized markup and quantity, 


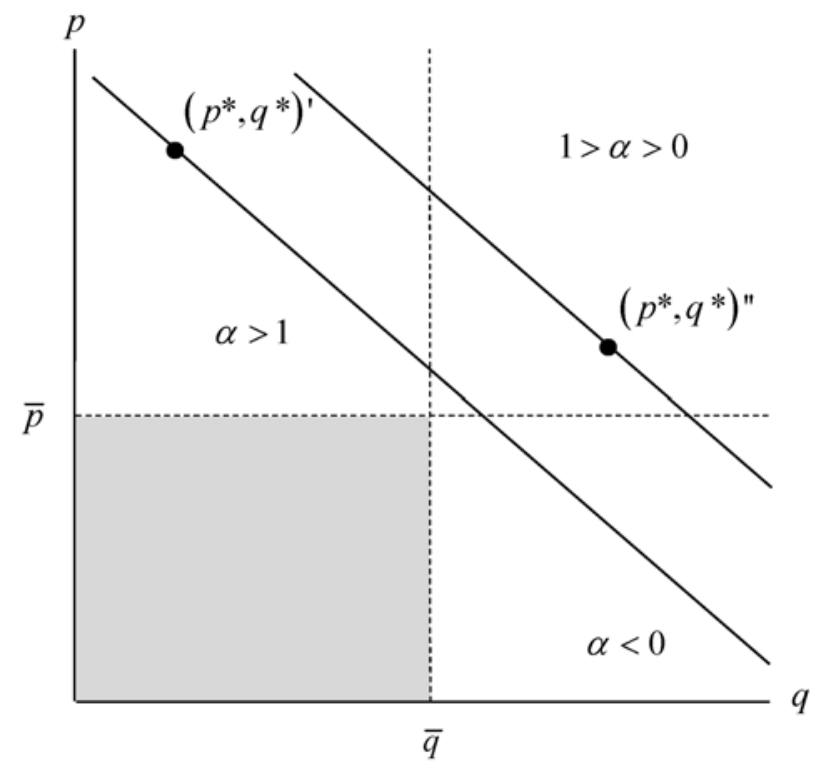

Figure 2: Iso total-shock functions: $\varepsilon>0$ given, $\alpha$ varies

assume that there is a composite shock $\varepsilon$ with $\varepsilon=\varepsilon(\mu, v)$, which translates into specific realizations of the optimized price, the corresponding unit costs, and the optimized quantity. Specifically, it is useful to write the optimized price, cost and quantity as $p^{*}=\kappa+\widehat{\alpha}_{1} \varepsilon, c^{*}=\bar{c}+\widehat{\alpha}_{2} \varepsilon$, and $q^{*}=\bar{q}+\widehat{\alpha}_{3} \varepsilon$, respectively, with $\widehat{\alpha}_{1}+\widehat{\alpha}_{2}+\widehat{\alpha}_{3}=1$. To simplify notation, the optimized markup and quantity can then be rewritten as:

$$
p^{*}-c^{*}=\bar{p}+\alpha \varepsilon, \text { and } q^{*}=\bar{q}+(1-\alpha) \varepsilon,
$$

respectively, with $\bar{p}=\kappa-\bar{c}$ and $\alpha=\widehat{\alpha}_{1}-\widehat{\alpha}_{2}$, where the type parameter $\alpha$ determines how a given total shock $\varepsilon$ is divided between the optimized markup and the optimized quantity. Consider Figure 1, again. Total shocks $\varepsilon^{\prime}$ and $\varepsilon^{\prime \prime}$ can be written as $\varepsilon^{\prime}=$ $\mathrm{I}^{\prime}+\mathrm{II}^{\prime}$ and $\varepsilon^{\prime \prime}=\mathrm{I}^{\prime \prime}+\mathrm{II}^{\prime \prime}$, respectively, where $\mathrm{I}^{\prime}+\mathrm{II}^{\prime}>\mathrm{I}^{\prime \prime}+\mathrm{II}^{\prime \prime}$ and $\alpha^{\prime}>1>\alpha^{\prime \prime}>0$ (because $\bar{p}<\left(p^{*}\right)^{\prime \prime}<\left(p^{*}\right)^{\prime}$ and $\left.\left(q^{*}\right)^{\prime \prime}>\bar{q}>\left(q^{*}\right)^{\prime}\right)$, which leads to $\left(p^{*}, q^{*}\right)^{\prime}=$ $\left(\bar{p}+\alpha^{\prime} \varepsilon^{\prime}, \bar{q}+\left(1-\alpha^{\prime}\right) \varepsilon^{\prime}\right)$ and $\left(p^{*}, q^{*}\right)^{\prime \prime}=\left(\bar{p}+\alpha^{\prime \prime} \varepsilon^{\prime \prime}, \bar{q}+\left(1-\alpha^{\prime \prime}\right) \varepsilon^{\prime \prime}\right)$.

With this formulation for optimized markup and optimized quantity, a positive shock $(\varepsilon>0)$ increases both the markup and quantity if the type-parameter $\alpha$ is between 0 and 1, i.e., $\alpha \in[0,1]$, while a positive shock increases the optimized markup and reduces the optimized quantity if the type-parameter is greater than 


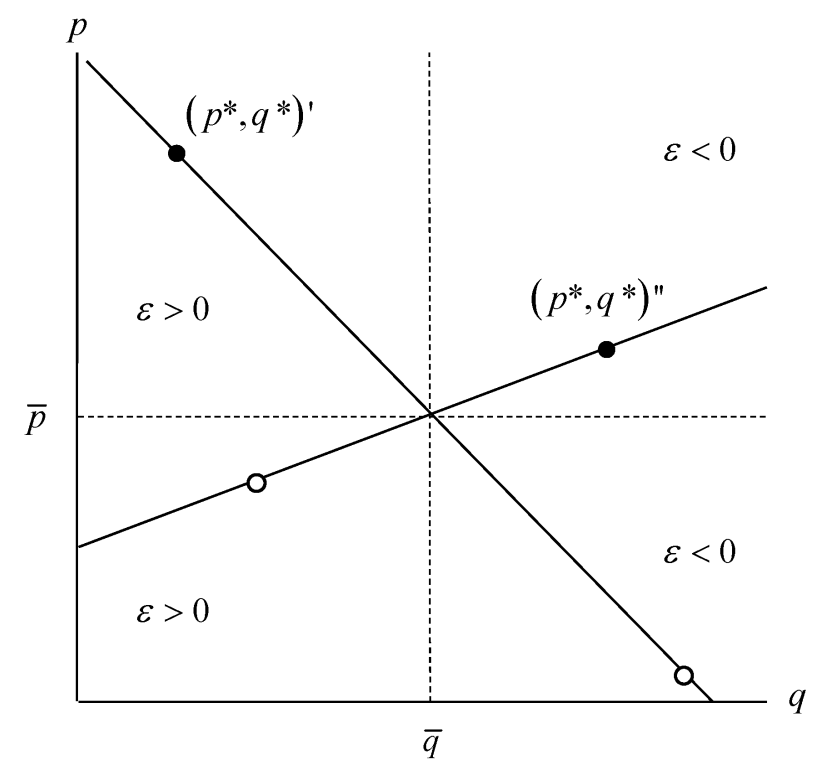

Figure 3: Iso-type functions: $\alpha$ given, $\varepsilon$ varies

1, and vice-versa if the type-parameter is negative. Figure 2 uses the price-quantity space to illustrate these three different cases and, what we call, the concept of isototal-shock functions (depicted as the solid lines). More specifically, iso-total-shock functions determine all the combinations of optimized markups and quantities that can be achieved for a given value of noise term $\varepsilon$ and with variations of the value of the type parameter $\alpha$.

Figure 2 displays iso-total-shock functions for shocks $\varepsilon^{\prime}>\varepsilon^{\prime \prime}>0$; so, both realizations of $\varepsilon$ are positive. The figure shows three unshaded areas: (i) the top-left area, (ii) the top-right area, and (iii) the bottom-right area. In the first, top-left area, the positive shock increases the optimized markup and reduces the optimized quantity relative to $\bar{p}$ and $\bar{q}$, respectively, which is true for $\alpha>1$. In the second, top-right area, the positive shock increases both the optimized markup and the optimized quantity, which is true for $0<\alpha<1$. In the third, top-bottom area, the positive shock reduces the optimized markup and increases the optimized quantity relative to $\bar{p}$ and $\bar{q}$, respectively, which is true for $\alpha<0$.

Alternatively, suppose that the type parameter is fixed, while the total shock $\varepsilon$ varies. This leads to, what we call, the concept of iso-type functions. More specifically, iso-type functions determine all the combinations of optimized markups 
and quantities that can be found for a given value of the type parameter $\alpha$ and with variations of the value of noise term $\varepsilon$. Figure 3 illustrates iso-type functions for $\alpha=\alpha^{\prime}, \alpha^{\prime \prime}$. For $\alpha=\alpha^{\prime}>1$, the iso-type function is a linear and downwardsloping line where a positive total shock increases the optimized markup relative to the expected optimized markup and reduces the optimized quantity relative to the expected optimized quantity, while the reverse is true for a negative total shock. The solid dot on the downward-sloping iso-type function, determines the optimized markup and quantity for $\varepsilon=\varepsilon^{\prime}$, while the open dot determines the optimized markup and quantity for $\varepsilon=-\varepsilon^{\prime}$. On the other hand, for $\alpha=\alpha^{\prime \prime} \in(0,1)$, the iso-type function is a linear and upward-sloping line where a positive total shock increases both the optimized markup and the optimized quantity relative to the expected optimized markup and the expected optimized quantity, respectively, while the reverse is true for a negative total shock. The solid dot on the upward-sloping iso-type function determines the optimized markup and quantity for $\varepsilon=\varepsilon^{\prime \prime}$, while the open dot determines the optimized markup and quantity for $\varepsilon=-\varepsilon^{\prime \prime}$. Note that, at this stage of the analysis, no assumptions are made on the distribution $\varepsilon$, except that this noise term has expectation zero, and that the variance exists and is given by $\sigma^{2}$. The two realizations of the noise terms for each population (altogether four realizations determined by the solid and open dots) are chosen for expositional purposes.

Note that only the dots will ultimately determine expected profits when there are no capacity constraints, while the iso-total-error and the iso-type functions just help to identify their locations. Furthermore, the shapes of the iso-total-error and the iso-type functions illustrate possible limitations of the present approach. For instance, we will show that the linear functional form for iso-type functions limits on the generality of some of our results. Still, we will discuss several examples in Section 3 to show that this way of modeling demand uncertainty captures a wide range of different functional forms for demands and costs and does help in identifying the consequences of uncertainty on monopoly profits. 


\section{3 (Unconstrained) Expected profit}

Letting $E$ denote the expectation operator and $\sigma$ the standard deviation of the total shock $\varepsilon$, expected profit, denoted $E \pi$, can be written as $E \pi=E\left[\left(p^{*}-c\right) q^{*}\right]$. Substituting $\bar{p}+\alpha \varepsilon$ for $p^{*}-c$ and $\bar{q}+(1-\alpha) \varepsilon$ for $q^{*}$, expanding and taking expectations yields:

$$
E \pi=\overline{p q}+\left(\alpha-\alpha^{2}\right) \sigma^{2}
$$

The right-hand side is the sum of two terms. The first term is a constant, while the second term is a quadratic function of the type-paramter, $\alpha$, and quadratic in the standard deviation $\sigma$. For this scenario, where the optimized markup and the optimized quantity can each be decomposed into two additive terms, where one is deterministic and the other is stochastic, equation (2) directly implies the main proposition of the present study: ${ }^{9}$

Proposition 1 Assume that the type parameter is constant in the total shock $\varepsilon$. A necessary and sufficient condition for an increase in uncertainty measured by the standard deviation parameter $\sigma$ to increase expected monopoly profit is that positive shocks $(\varepsilon>0)$ increase both the monopoly markup and the monopoly quantity, while negative shocks $(\varepsilon<0)$ reduce both of these values.

Figures 4 and 5 illustrate this proposition. Figure 4 depicts the profits associated with shock-type pairs $(\varepsilon, \alpha)=\left(\varepsilon^{\prime \prime}, \alpha^{\prime \prime}\right),\left(-\varepsilon^{\prime \prime}, \alpha^{\prime \prime}\right)$. It shows the profit gain in the case of a positive shock, $I+I I+3 a$, and the profit loss in the case of a negative shock, $I+I I+a$, relative to the profit when shocks are zero, $\overline{p q}$, with positive difference $2 a$. Thus, an increase in uncertainty measured by the standard deviation of the total shock, $\sigma$, increases expected profit in this situation with $\alpha=\alpha^{\prime \prime}$. The picture changes for the situation depicted in Figure 5 with shock-type pairs $(\varepsilon, \alpha)=\left(\varepsilon^{\prime}, \alpha^{\prime}\right),\left(-\varepsilon^{\prime}, \alpha^{\prime}\right)$. The profit gain in the case of a positive shock is $I-I I-a$, and the profit loss in the case of a negative shock is $I+a-I I$, relative to profit $\overline{p q}$. Subtracting this loss from

\footnotetext{
${ }^{9}$ The results stated in Proposition 1 are independent of whether the type parameter $\alpha$ defines the differences between actual and expected values of optimized price markups and optimized quantities in absolute values, as is done by the present definition of uncertainty types, or in relative terms (i.e., for $p^{*}-c^{*}=\bar{p}(1+\alpha \varepsilon)$ and $q^{*}=\bar{q}(1+(1-\alpha) \varepsilon)$ ).
} 


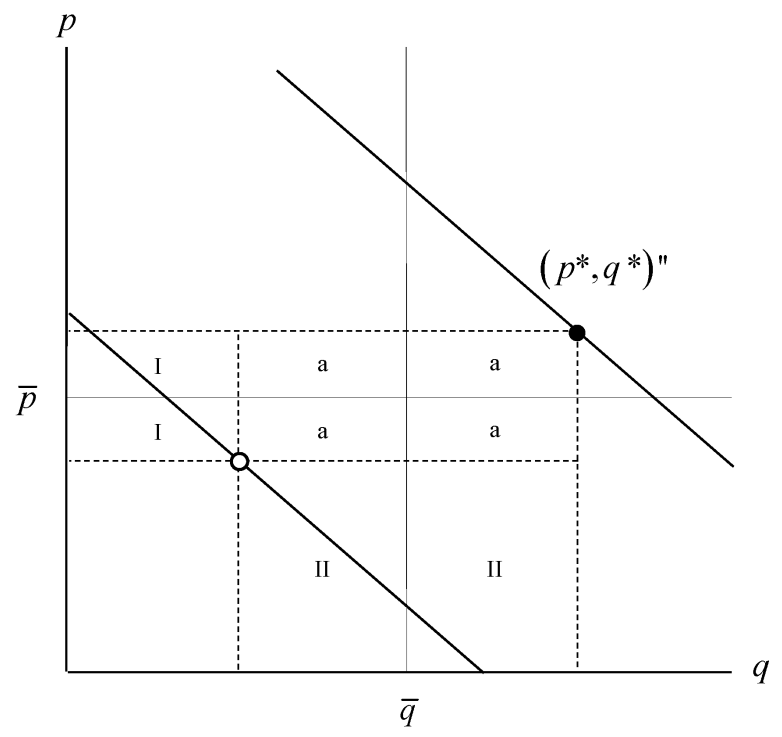

Figure 4: Expected profit gain (relative to certainty outcome $\overline{p q}$ ) is $2 a$ when the type parameter is (strictly) between 0 and 1

the gain we find a net loss of $2 a$. Thus, an increase in $\sigma$ reduces expected profit in this situation with $\alpha=\alpha^{\prime}$. A similar situation emerges for a type-parameter strictly less than 0 .

Proposition 1 assumes that the type parameter is constant in $\varepsilon$. If instead the type parameter is a function of $\varepsilon$ (e.g., $\alpha=\varepsilon$ ), the picture changes. First, in this situation an increase in uncertainty measured by the standard deviation $\sigma$ may increase expected profit even when, for specific values of $\varepsilon$, the type parameter is negative or exceeds 1 . Second, the overall effect of uncertainty on expected profit (which is maximized for $\alpha=1 / 2$ when the type parameter is constant in $\varepsilon$ ) depends largely on the distribution of $\varepsilon$. Appendix A discusses an example where uncertainty types are a function of $\varepsilon$, i.e., $\alpha=\varepsilon$, to illustrate these cases.

\section{Identification}

This section assumes specific functional forms and identifies parameters such that the monopoly markup and quantity are of the specific additive structure discussed above. The main lesson of this part is that the same type of uncertainty can arise from a 


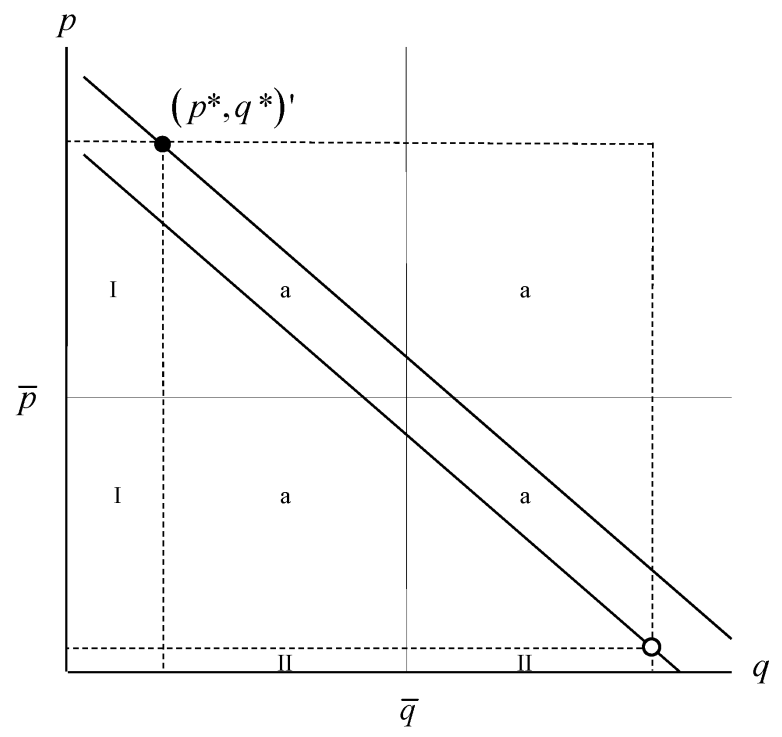

Figure 5: Expected profit gain is $-2 a$ (relative to certainty outcome $\overline{p q}$ ) when the type parameter is (strictly) greater than 1

range of utility and cost functions; consequently, the choice of specific functional forms may often be without loss of generality. The functional forms discussed in this section will further be used to analyze the joint effect of uncertainty types and functional forms on monopoly capacity choice in Section 4.

\subsection{Stochastic utility and zero or deterministic cost}

Linear utility. Utility is $B=\phi q$ for $q \leq \psi$ and zero otherwise; costs are normalized to zero, i.e., $c=0$. In this scenario monopoly markup and quantity are directly determined by parameters $\phi$ and $\psi$, respectively. If parameters are stochastic with $\phi=\bar{p}+\alpha \varepsilon$ and $\psi=\bar{q}+(1-\alpha) \varepsilon$, this is perhaps the most simple utility and cost structure that can be used to analyze the uncertainty types of the form developed above.

Quadratic utility. Utility is $B=a q-b q^{2} / 2$ and $c=0$, which leads to the inverse demand $p=a-b q$, monopoly price $a / 2$ and monopoly quantity $a /(2 b)$. In this scenario, stochastic parameters $a=2(\bar{p}+\alpha \varepsilon)=2 p^{*}$ and $b=(\bar{p}+\alpha \varepsilon) /(\bar{q}+(1-\alpha) \varepsilon)=$ $p^{*} / q^{*}$ can be used to analyze uncertainty types. More specifically, for the two special 
cases with $\alpha=0$ and $\alpha=1$, parameters can be written as

$$
(a, b)=\left\{\begin{aligned}
(2 \bar{p}, \bar{p} /(\bar{q}+\varepsilon)) & \text { for } \quad \alpha=0 \\
(2(\bar{p}+\varepsilon),(\bar{p}+\varepsilon) / \bar{q}) & \text { for } \quad \alpha=1 .
\end{aligned}\right.
$$

The discussion of these two special cases illustrates how identification can be used to provide a better understanding of the conditions that lead to stochasticity in the monopoly price or quantity. For instance, if demand is linear with a deterministic choke price, $a=2 \bar{p}(\Leftrightarrow \bar{p}=a / 2)$, the monopoly price is deterministic, while the monopoly quantity is stochastic. With linear demands, the price-elasticity of demand can be written as $-p /(a-p)$, which shows that the price-elasticity of demand is deterministic when $a$ is deterministic. (Since the monopoly price is determined by the inverse elasticity of demand when operating costs are normalized to zero, this explains why the monopoly price is deterministic in this special case $(\alpha=0)$.) On the other hand, if demand is linear with a stochastic choke price, $a=2(\bar{p}+\varepsilon)$, and stochastic demand slope, $b=(\bar{p}+\varepsilon) / \bar{q}$, linear demand can be written as $p=(\bar{p}+\varepsilon)(2-q / \bar{q})$, which implies that the saturation demand is implicitly determined by $0=(\bar{p}+\varepsilon)(2-q / \bar{q})(\Leftrightarrow q=2 \bar{q})$ and the monopoly quantity is deterministic (with linear demands and zero marginal cost, the monopoly quantity is half the saturation demand).

Power utility. Utility now is $B=u q^{\gamma}$. This leads to the inverse demand $p=$ $u / q^{1-\gamma}$ and constant price elasticity of demand $-1 /(1-\gamma)$. For this functional form, existence of a monopoly solution requires positive marginal cost $c$, which are assumed to be constant and deterministic in this example. Furthermore, $\gamma<1$ is required to ensure strict concavity of demand. Altogether, this leads to the monopoly price $c / \gamma$ and monopoly quantity $\left(c /\left(u \gamma^{2}\right)\right)^{-1 /(1-\gamma)}$. Stochastic parameters

$$
\gamma=\frac{c}{p^{*}}, u=\frac{1}{c}\left(q^{*}\right)^{\frac{p^{*}-c}{p^{*}}}\left(p^{*}\right)^{2}
$$

imply uncertainty types of the above mentioned form. For the two special cases, parameters can be written as

$$
(\gamma, u)=\left\{\begin{array}{r}
\left(c /(c+\bar{p}),(\bar{q}+\varepsilon)^{\frac{\bar{p}}{c+\bar{p}}}(c+\bar{p})^{2} / c\right) \text { for } \quad \alpha=0 \\
\left(c /(c+\bar{p}+\varepsilon), \bar{q}^{\frac{\bar{p}+\varepsilon}{c+\bar{p}+\varepsilon}}(c+\bar{p}+\varepsilon)^{2} / c\right) \text { for } \quad \alpha=1 .
\end{array}\right.
$$


The price elasticity of demand and thus the monopoly price is deterministic if $\gamma=$ $c /(c+\bar{p})$, which explains why this corresponds to a situation with $\alpha=0$. On the other hand, if $\gamma=c /(c+\bar{p}+\varepsilon)$, the price elasticity of demand is stochastic, which means that the monopoly price is stochastic, while the scaling parameter $u$ is also stochastic and determined in such a way that the monopoly quantity remains constant.

\subsection{Stochastic utility and stochastic cost}

Operating costs can be highly uncertain. For example, production costs may be largely determined by the prices for natural resources, fuel or by exchange rates all of which can be fluctuating quite strongly. This section concentrates on a quadratic utility function of the form discussed above in order to illustrate that uncertainty types capture stochasticity in the demand and cost functions.

Linear cost. Assume that costs are linear and given by $w q$, which leads to marginal costs $c=w$. Parameters $a-w=2\left(p^{*}-c^{*}\right)$ and $b=\left(p^{*}-c^{*}\right) / q^{*}$ with $c^{*}=w$ lead to uncertainties of the types developed in Section 2. Note that only the difference between the choke price, $a$, and unit cost, $w$, is relevant for the result. Thus, for the monopolist it is equivalent, under these conditions with constant unit costs, whether the choke price is uncertain, the unit costs are uncertain, or both the choke price and the unit costs are uncertain.

Quadratic cost. Assume that costs are quadratic and given by $w q^{2}$. This leads to $c=w q$; thus, average costs are increasing in output, and parameters $a=2\left(p^{*}-c^{*}\right)$ and $w+b=\left(p^{*}-c^{*}\right) / q^{*}$ lead to the above mentioned uncertainty types. This shows, that in this case, only the sum $(w+b)$ of the shift parameter for costs and the absolute value of the demand slope is relevant for the monopolist.

Altogether this shows that uncertainty types are not tied to specific functional forms of utilities, demands or costs. Assumptions about the corresponding functional forms may therefore not be as limiting as it may seem at first view, while implicit or explicit assumptions about the type of uncertainty can have quite strong effects 
on expected monopoly profit.

\section{Capacity Choice}

The main purpose of this section is to demonstrate how uncertainty types can affect the monopoly firm's behavior. To do this, we explore the role of uncertainty types for the monopoly's incentives to invest in capacity. Think of, for example, airports where the quantity of flights may be constrained by airport slots, which entitle airlines to make use of airport capacity at a specific point in time. Airport slots are typically determined by runway capacity, which in turn would depend on the airport's incentives to invest in infrastructure. ${ }^{10}$ Other examples are airlines or train operating companies where transport capacity depends on vehicle sizes. Nontransport related examples are universities, schools, fire fighters and hospitals where capacity is determined by the number and/or size of facilities, or the number of workers.

\subsection{One-dimensional capacity}

Assume that output is constrained by capacity, denoted $k$, with $q=\min \{k, \bar{q}+(1-\alpha) \varepsilon\}$.

The monopoly firm can invest in capacity with convex costs, denoted $g$ with $g=g(k)$ and $g^{\prime}>0$, but does so before knowing the exact realization of the noise term $\varepsilon$. To ensure the existence of a solution, assume that $\omega(g(k))$, is sufficiently high. Assume, here and in the remainder of this section, that the noise term follows a Bernoulli

\footnotetext{
${ }^{10}$ Most airports in Europe are slot constrained, while capacity allocation is based on the firstcome-first-serve principal in the US (e.g., Czerny et al., 2008). During the last several years airlines and passengers have been suffering from runway congestion and delays at busy airports, and airport delays have become a major public policy issue. Airports have traditionally been owned by governments, national or local, owing partly to their exhibiting many features of a local monopoly. This is changing, however: many airports around the world have been, or are in the process of being, privatized. Taken together, todays shortage of airport capacity makes the examination of airport capacity choice (and related congestion tolls) an important and active research area in recent years; see, e.g., Zhang and Czerny (2012) for a comprehensive literature review.
} 
distribution with $\varepsilon \in\{-\sigma, \sigma\}$, where the probalities of positive and negative shocks are the same and given by $1 / 2$ in order to ensure that expected noise is zero, i.e., $E[\varepsilon]=0 .{ }^{11}$ Anticipating that the capacity constraint is binding only in the case of a positive shock (thus, not binding in the case of a negative shock, which rules out that $\alpha>1$, where a negative shock would lead to an increase in quantity), price and quantity in the negative shock state are given by $\bar{p}-\alpha \sigma$ and $\bar{q}-(1-\alpha) \sigma$, respectively, and thus independent of capacity. ${ }^{12}$ On the other hand, in the positive shock state price and quantity are determined by capacity and given by $B^{\prime}(k ; \alpha, \sigma)$ and $k$, respectively. Since the positive- and negative-shock states occur with the same probability of $1 / 2$, expected profit can be written as

$$
E \pi=\frac{1}{2} \cdot(\underbrace{(\bar{p}-\alpha \sigma)(\bar{q}-(1-\alpha) \sigma)}_{\varepsilon=-\sigma}+\underbrace{\left(B^{\prime}(k ; \alpha, \sigma)-c\right) \cdot k}_{\varepsilon=\sigma})-g(k) .
$$

The first term inside the parentheses is the revenue when the shock is negative and the second term is the revenue when the shock is positive and when output is limited from above by capacity $k$.

The optimal capacity is determined by the first-order condition $\partial E \pi / \partial k=0$, which can be written as

$$
\frac{1}{2}(1+\omega(B(k ; \alpha, \sigma))) \cdot B^{\prime}(k ; \alpha, \sigma)-\left(c+g^{\prime}\right)=0 .
$$

The left-hand side shows that the investment incentives depend on how the type parameter and the standard deviation $\sigma$ affect the marginal utility and the curvature of the utility function. For instance, in the case of the linear utility, $\omega(B(k ; \alpha, \sigma))=0$ and $B^{\prime}(k ; \alpha, \sigma)=\bar{p}+\alpha \sigma$ in optimum, where the latter shows that the investment

\footnotetext{
${ }^{11} \mathrm{~A}$ similar timing and distribution of the noise term has been analyzed by Proost and van der Loo (2010).

${ }^{12}$ Capacity may be binding in the low-demand state if capacity cost are high, i.e., optimal capacity is sufficiently small. This is because marginal cost are zero in the low demand state, which leads to a low monopoly price relative to the high-demand state where marginal costs are positive and determined by marginal capacity cost. This is similar to the so-called "backhaul" issue discussed by, e.g., Rietveld and Roson (2002). We abstract away from the case where capacity is binding in the high- and low-demand states by assuming that marginal capacity costs are sufficiently low or uncertainty measured by the standard deviation $\sigma$ is sufficiently high.
} 
incentives associated with an increase in uncertainty increase in the type parameter, i.e., when the optimized price markups become more uncertain relative to optimized quantities.

For an intuition, consider a situation where the type parameter is zero. The optimal price is deterministic in this situation but also the quantity in the positive shock case is deterministic and given by $k$; thus, even though the optimal unconstrained monopoly quantity is stochastic, the outcome in the positive shock state is independent of the standard deviation parameter $\sigma$ and so are the investment incentives. For a strictly positive type parameter, the quantity in the positive shock state is still given by $k$ and thus deterministic, but the markup increases in the standard deviation, which means that investment incentives increase in uncertainty measured by $\sigma .^{13}$

For the quadratic utility, the first term on the right-hand side of the first-order condition (7), $(1+\omega(B(k ; \alpha, \sigma))) \cdot B^{\prime}(k ; \alpha, \sigma)$, can be written as $2 p^{*} \cdot\left(q^{*}-k\right) / q^{*}$, where $q^{*}-k>0$ in optimum. If a positive shock increases both the monopoly price and quantity, i.e., for $\alpha \in[0,1]$, this term is strictly increasing in the standard deviation $\sigma$. On the other hand, the effect of uncertainty types on the investment incentives can work into different directions. To see this, take the first partial derivate of the first term on the right-hand side with respect to the type parameter which yields $2 \sigma / q^{*} \cdot\left(q^{*}-k-p^{*} k / q^{*}\right)$, which is negative for sufficiently high values of the monopoly price in positive shock state, i.e., $p^{*}>\left(\left(q^{*}\right)^{3}-k\right) /\left(k q^{*}\right)$, and positive otherwise.

For the power utility, the first term on the right-hand side of the first-order condition (7) can be written as $c \cdot\left(q^{*} / k\right)^{\left(p^{*}-c\right) / p^{*}}$. Totally differentiating the firstorder condition (7) with respect to capacity $k$ and standard deviation parameter $\sigma$ reveals that also this expression is increasing $\sigma$. Thus, also for the quadratic

\footnotetext{
${ }^{13}$ With linear utility, the result that investment incentives are an increasing function of the type parameter depends on the level of capacity cost. If capacity cost are low, it may be optimal to choose capacity equal to the optimal quantity $q^{*}$ in the high demand state. Since $q^{*}$ is a decrasing fucntion of the type parameter in this situation (i.e., $\left.q^{*}=\bar{q}+(1-\alpha) \sigma\right)$ investment incentives are decreasing in the type parameter under these special condiitons with linear utility and low capacity costs.
} 
and power utilities it holds that an increase in uncertainty measured by the standared deviation parameter increases capacity investments. Furthermore, the first partial derivate of the first term on the right-hand side with respect to the type parameter yields a strictly negative mathematical expression, which can be written as $\left(-\left(p^{*}-c\right) p^{*}+c q^{*} \log \left(q^{*} / k\right)\right)\left(c \sigma k^{\left(p^{*}-c\right)}\left(q^{*}\right)^{c} /\left(p^{*}\right)^{2}\right)^{-1 / p^{*}}$. Thus, an increase in the type parameter reduces investment incentives in the power utility case.

Altogether this shows that an increase in the type parameter can have positive or a negative effect on capacity investments, which relies heavily on the employed functional forms.

\subsection{Two-dimensional capacity}

While it is common to treat capacity as one-dimensional, the capacity concept is usually more complex in reality. Think of airlines, train operating companies or busses where capacity is determined by both the quantity as well as the size of vehicles. Or, think of hospitals, firefighters, schools and universities, where capacity depends on the number and size of facilities. With multi-dimensional capacity, one may wonder how uncertainty types may affect the optimal composition of capacity. To analyze this, assume that capacity is determined by the product of the number of vehicles or facilities, denoted $f$, and the size of vehicles or facilities, denoted $s$, which implies $k=s f$. Costs are still a convex function of capacity $k$. For example, administration costs and land costs may depend on the overall capacity and not on the composition of capacity. In addition, there is a given cost, denoted $d$ with $d>0$, per unit of $f .{ }^{14}$ This leads to (assured strictly convex) cost $d f+g(s f)$.

Another feature is that the quality of the product or service can be related to $f$. This is true in transport because passengers are more likely to travel at their preferred time when more vehicles are in operation (e.g., Douglas and Miller, 1974). Or, facilities are in average more conveniently located for customers if they are more densely spread across a city or country. To capture this, we assume that the

\footnotetext{
${ }^{14}$ For $d=0$, there is no solution because in this case it is optimal to choose an arbitrarily small seat capacity and increase frequency supply to infinity because frequency supply increases demand, which is not true for seat capacity.
} 
generalized price of consumption is the sum of the price, $p$, and a second term, denoted $\Gamma$, which captures service quality and is a decreasing and strictly convex function of quantity $f$, i.e., $\Gamma=\Gamma(f)$ with $\Gamma^{\prime}<0$ and $\Gamma^{\prime \prime}>0$. In demand equilibrium the generalized price is equal to the marginal utility of consumption: $p+\Gamma=B^{\prime}$ or equivalently $p=B^{\prime}-\Gamma$, where the right-hand side of the latter term determines the markup on constant and zero marginal costs. The latter shows that $\Gamma$ can have the interpretation of per-unit cost to the company and that the framework developed above can be used to analyze the quality effects of $f$ by assuming $p=B^{\prime}$ and $c=\Gamma$.

Assuming that the capacity constraint is binding only in the case of a positive shock (i.e., $\bar{q}+\sigma>s f$ in optimum), expected profit can be written as

$$
E \pi=\frac{1}{2}\left((\bar{p}-\alpha \sigma)(\bar{q}-(1-\alpha) \sigma)+\left(B^{\prime}(s f ; \alpha, \sigma)-\Gamma(f)\right) \cdot s f\right)-d f-g(s f) .
$$

Note that $\partial(\bar{p}-\alpha \sigma) / \partial f=-\Gamma^{\prime}$ reflecting the fact that a higher $f$ also raises fare in the low state of demand, which means that the first-order conditions that determine optimal capacity can be written as

$$
\begin{aligned}
& \frac{\partial E \pi}{\partial s}=\left(\frac{B^{\prime}(s f ; \alpha, \sigma)-\Gamma(f)}{2}-g^{\prime}\right) f=0, \\
& \frac{\partial E \pi}{\partial f}=\underbrace{\left(\frac{B^{\prime}(s f ; \alpha, \sigma)-\Gamma(f)}{2}-g^{\prime}\right)}_{=0} s-\frac{\Gamma^{\prime}}{2}(\bar{q}-(1-\alpha) \sigma+s f)-d=0 .
\end{aligned}
$$

The first-order condition (9) implies that the optimal capacity $k$ ensures that the marginal capacity costs, $g^{\prime}$, are equal to the price that can be charged in the case of a positive shock, $B^{\prime}(s f ; \alpha, \sigma)-\Gamma$, evaluated at the probability of the positive shock to occur, $1 / 2$. Since the capacity constraint is binding only in the state of a positive shock, it is intuitive that only the price under the conditions of a positive shock are relevant for the capacity choice. The same logic applies to the capacity effect of $f$, which is why the first term on the right-hand side of (10) is zero in optimum. The second term on the right-hand side of (10) measures the expected quality gain associated with the marginal increase in $f$, which is equal to the marginal cost of $f$ in optimum.

To see how the optimal composition of capacity depends on the uncertainty type, it is useful to define a critical value of the type-parameter, denoted $\widetilde{\alpha}$ with 
$\widetilde{\alpha} \equiv \Gamma^{\prime} /\left(\Gamma^{\prime}-s\right)$, where the right-hand side is strictly positive and strictly less than 1. Furthermore, it is useful to impose an assumption on the shape of the capacity cost function $g$, which ensures that size $s$ and quantity $f$ are substitutes in the sense that an increase of one variable reduces the expected benefits of an increase in the other variable, i.e., $\partial^{2} E \pi / \partial s \partial f<0:^{15}$

Assumption 1 The capacity cost function is sufficiently convex in the sense that $\omega(g(k))>-f \Gamma^{\prime} /\left(2 g^{\prime}(k)\right)$.

Totally differentiating the first-order conditions (9) and (10) and applying Cramer's rule yields the following results (the proof is relegated to Appendix B):

Proposition 2 If the capacity cost function is sufficiently convex in the sense made precise by Assumption 1, and if quantity $q$ is sufficiently uncertain in the sense that $\alpha<\widetilde{\alpha}$, an increase in uncertainty measured by the standard deviation parameter $\sigma$ increases optimal size $s$ and reduces optimal quantity $f$.

This proposition is useful to show how uncertainty types can affect the composition of capacity $k$. While an increase in uncertainty always increases the marginal benefit of an increase in size $s$, the marginal benefit of an increase in quantity $f$ is clear-cut and negative in sign for sufficiently uncertain quantity $q$ in the sense that $\alpha<\widetilde{\alpha}$. For an intuition, recall that the revenue gains of investment in quantity $f$ depend heavily on the quantity $q$. Furthermore, if $q^{*}$ is small in the negative shock state (when the type parameter takes a low value in the sense that $\alpha<\widetilde{\alpha}$ ), this reduces the incentives to invest in $f$ although $q^{*}$ is high in the positive shock case because quantity is constrained by capacity in the positive shock state and thus $q^{*}$ does not become effective in the positive shock state. Proposition 2 is silent about the effect of demand uncertainty on $s$ and $f$ when $q$ is not sufficiently uncertain (i.e., $\alpha>\widetilde{\alpha}$ ) because it is difficult to derive general insights on the effect of demand uncertainty on capacity choice under these conditions. To shed some further light on the effect of uncertainty on the optimal composition of and level of capacity $k$ and expected profit for the entire range of the type-parameter, the following numerical example is used. 


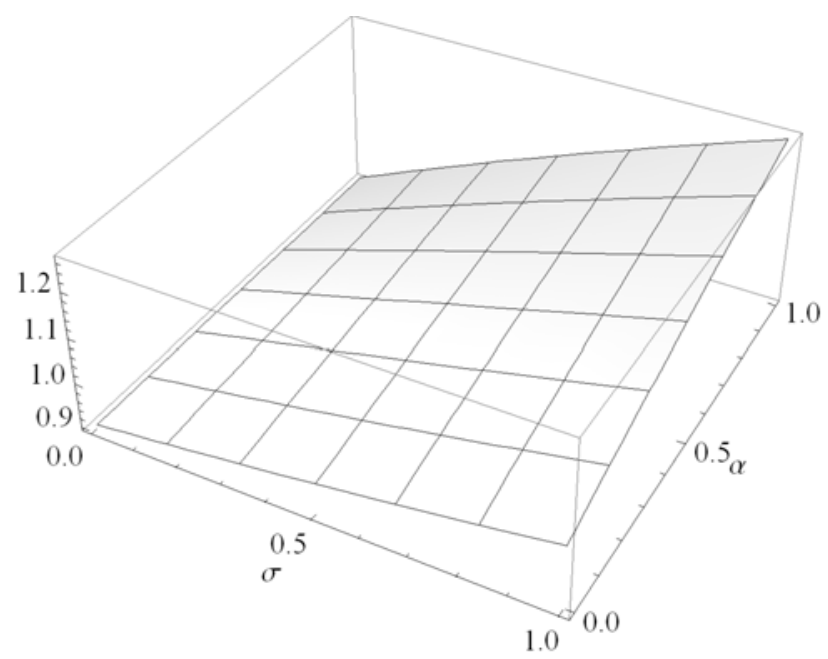

Figure 6: Optimal size $s$

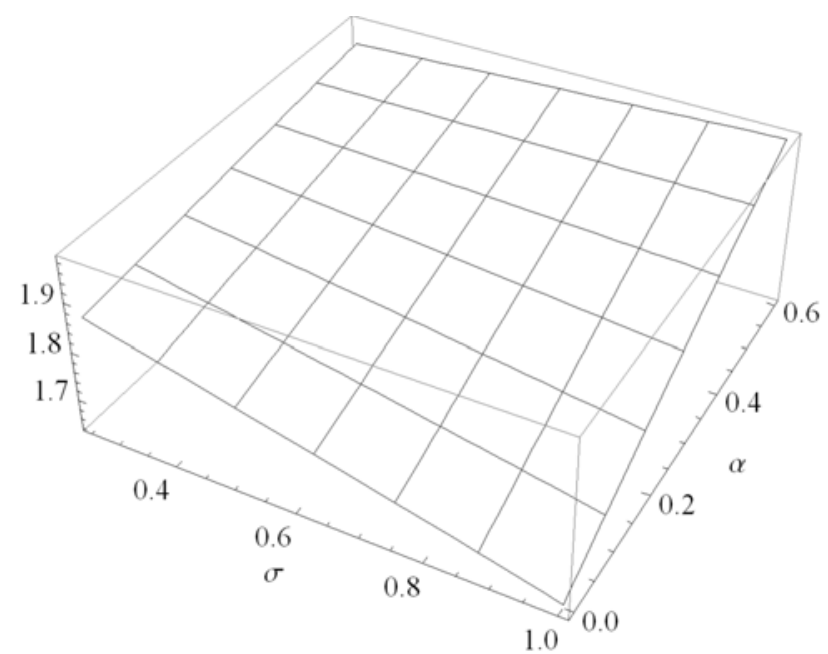

Figure 7: Optimal quantity $f$ 


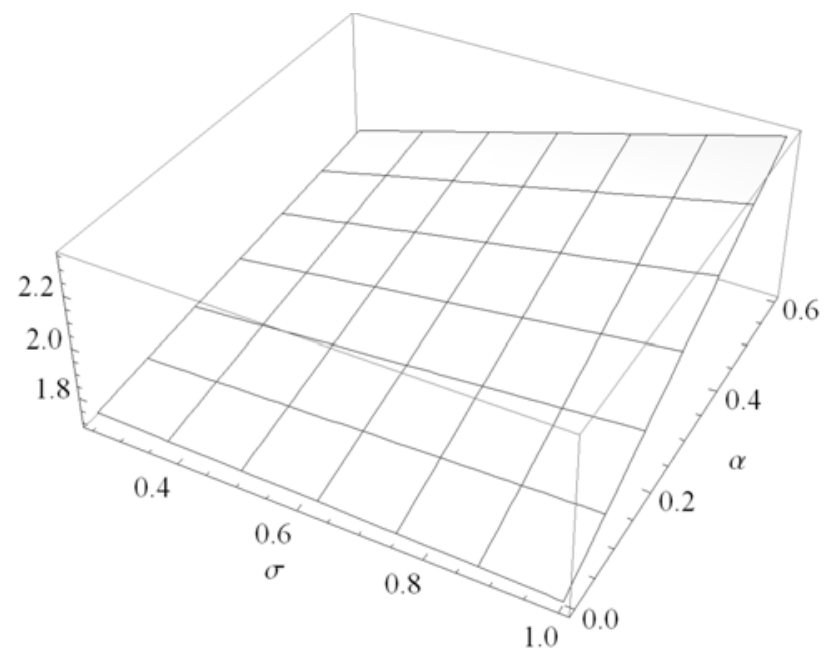

Figure 8: Optimal overall capacity $s f$

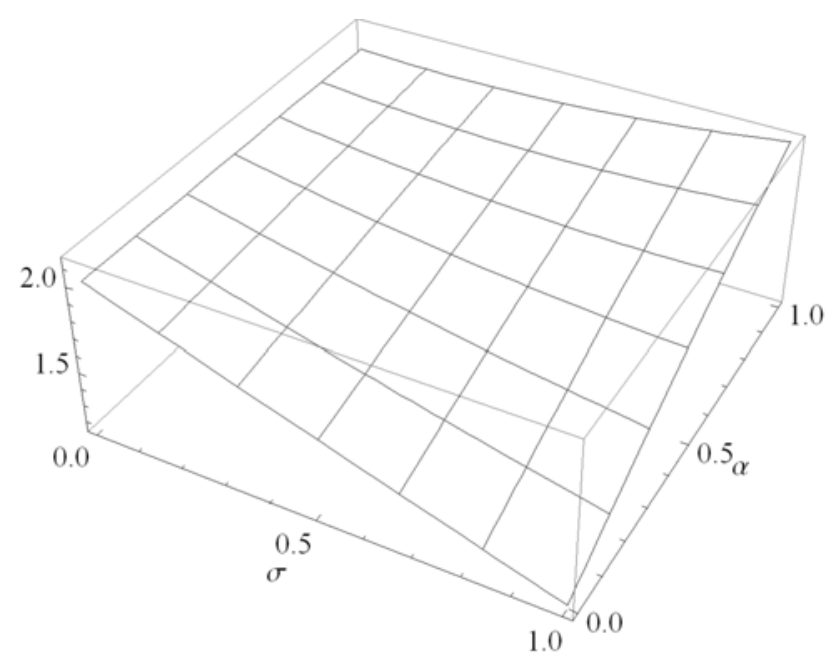

Figure 9: Expected profit, $E \pi$, when capacity is two-dimensional 
Figures 6 to 9 display the optimal values of size $s$, quantity $f$, capacity $s f$ and the corresponding optimized expected profit $E \pi$, respectively, depending on the typeparameter and uncertainty measured by $\sigma$. The diagrams are derived for linear utility, the functional form $\Gamma=\gamma / f$ and parameter values $\bar{p}=\bar{q}=2, \gamma=1 / 2$, $d=1 / 4$ and $r=1 .{ }^{16}$ Figure 6 shows that an increase in uncertainty is associated with an increase in the optimal size $s$, which is true for the entire range of the type parameter, while the relationship between uncertainty and the optimal quantity $f$ depends strongly on the value of the type-parameter: Figure 7 shows that an increase in uncertainty can be associated with an increase or a reduction in the optimal value of $f$ : If $q$ can be relatively well predicted and correspondingly monopoly price $p^{*}$ is more uncertain, the optimal value of $f$ increases in uncertainty, while it decreases in uncertainty otherwise. Figure 8 shows that total capacity can be decreasing in uncertainty for low values of the type parameter (for instance, this is true for $\alpha=0$ ), which means that the possibly negative effect of uncertainty on frequency supply can dominate the corresponding positive effect on vehicle sizes. Furthermore, since a low value of the type parameter means that $q^{*}$ is high in the positive shock state, while it does not become effective because of the capacity constraint, expected profit increases in uncertainty if the type parameter takes a sufficiently high value as illustrated in Figure 9. Altogether this shows that the uncertainty-type has a strong influence on the optimal composition of capacity and the effect of uncertainty on the total capacity and expected profit.

\section{Conclusions}

This paper has analyzed a framework where firms choose prices under perfect information, while capacity choices must be made under uncertainty about demand and cost conditions. A classification of continuous uncertainty types was proposed according to the effect of uncertainty on optimized monopoly markups and quantities. The main part of the analysis concentrated on situations where the ratio of

\footnotetext{
${ }^{15}$ This assumption will be satisfied in the numerical examples considered below.

${ }^{16}$ The functional forms, $\Gamma=\gamma / f$, are commonly used in the airline literature to capture the effect of frequency supply on schedule delays (e.g., Brueckner, 2004).
} 
the differences between the actual and expected values for optimized markups and quantities can be described by a (deterministic) constant value. It was shown that this framework is sufficiently general to capture a range of commonly used functional forms for utilities and costs. There are two main results of the analysis: first, an increase in uncertainty increases the expected monopoly profit only if a higher than the expected optimized markup is associated with a higher than the expected optimized quantity and vice-versa (when there are no capacity constraints). Second, the optimal level of capacity as well as the optimal composition of capacity in terms of the number and size of production units depend crucially on the type of uncertainty and the employed functional forms for utilities. Altogether, this shows that a precise knowledge of the uncertainty types is crucial for the derivation and understanding of monopoly investments in capacity.

The analysis provides several avenues for future research. While this paper used the monopoly example to develop and describe the theory of continuous uncertainty types, the concept of uncertainty types can be used to derive new insights in other contexts as well. For instance, consider a welfare example where the policy maker's problem is to internalize cost externalities. In this situation, the policy maker can choose between taxation and consumption limits as means for internalization (amongst other measures). Under uncertain market conditions, this is similar to the classical "prices vs. quantities" problem first analyzed by Weitzman (1974), where he found that the relative benefits of taxation (prices) or consumption limits (quantities) are determined by the relative slopes of demand and marginal external cost functions and how these functions are correlated. ${ }^{17}$ However, this analysis employs the rather strong assumption that slopes are deterministic. In this context with externalities, the distinction between uncertainty types can offer a different view on the issue. For instance, it is intuitive that prices should be preferred from the social viewpoint if the welfare-optimal price is deterministic in an otherwise uncer-

\footnotetext{
${ }^{17}$ Czerny (2010) showed that prices may be preferred over quantities in airline markets because the demand slope may always exceed the one of the marginal external congestion costs when linear functional forms are considered and because demand and external marginal congestion cost may be negatively correlated.
} 
tain environment, or quantities should be preferred if the welfare-optimal quantity is deterministic in an otherwise uncertain environment. Appendix $\mathrm{C}$ provides an analytical example to illustrate how uncertainty types can be used to analyze this kind of welfare problems.

The present study has only briefly discussed the case where the ratio of the differences between the actual and expected values for optimized markups and quantities are not deterministic (i.e., uncertainty types are varying in noise). However, this brief discussion already demonstrated that some of the results derived for constant uncertainty types do not carry over to the case of varying uncertainty types, which indicates that a more in-depth analysis of the varying uncertainty-types case is necessary to derive a more complete picture of the role of uncertainty types for economic behavior. Since the paper is theoretical in nature, it would also be useful to derive a more precise empirical understanding of the relevance of specific uncertainty types in reality. 


\section{References}

Abel, A. B. (1983), Optimal investment under uncertainty, American Economic Review 73: 228-233.

Aguirre, I., Cowan, S. and Vickers, J. (2010), Monopoly price discrimination and demand curvature, American Economic Review 100: 1601-1615.

Arnott, R., de Palma, A. and Lindsey, R. (1996), Information and usage of freeaccess congestible facilities with stochastic capacity and demand, International Economic Review 37: 181-203.

Arrow, K. J., Harris, T. and Marschak, J. (1951), Optimal Inventory Policy, Econometrica 19: 250-272.

Bagnoli, M. and Bergstrom, T. (2005), Log-concave probability and its applications, Economic Theory 26: 445-469.

Baron, D. P. (1970), Price uncertainty, utility, and industry equilibrium in pure competition, International Economic Review 11: 463-480.

Brown, G, Jr. and Johnson, M. B. (1969), Public utility pricing and output under risk, American Economic Review 59: 119-128.

Brueckner, J. K. (2004), Network structure and airline scheduling, Journal of Industrial Economics 52: 291-312.

Czerny, A. I. (2010), Airport congestion management under uncertainty, Transportation Research Part B 44: 371-380.

Czerny, A. I., Forsyth, P., Gillen, D. W., Niemeier, H.-M. (eds.) (2008), Airport Slots: International Experiences and Options for Reform, Ashgate Studies in Aviation Economics and Management, Ashgate.

Douglas, G. W. and Miller, J. C. (1974), Quality competition, industry equilibrium, and efficiency in the price-constrained airline market, American Economic Review 64: $657-669$. 
D'Ouville, E. L. and McDonald, J. F. (1990), Effects of demand uncertainty on optimal capacity and congestion tolls for urban highways, Journal of Urban Economics 28: $63-70$.

Dvoretzky, A., Kiefer, J. and Wolfowitz, J. (1952a), The inventory problem: I. Case of known distributions of demand, Econometrica 20: 187-222.

Dvoretzky, A., Kiefer, J. and Wolfowitz, J. (1952b), The inventory problem: II. Case of unknown distributions of demand, Econometrica 20: 450-466.

Gallego, G. and van Ryzin, G.J. (1994), Optimal dynamic pricing of inventories with stochastic demand over finite horizons, Management Science 40: 999-1020.

Hartman, R. (1972), The effects of price and cost uncertainty on investment, Journal of Economic Theory 5: 258-266.

Karlin, S. and Carr, C. (1962), Prices and Optimal Inventory Policy, in Arrow, K. et al. (eds.), Studies in Applied Probability and Management Science, Stanford University Press.

Khouja, M.J. (2000), Optimal ordering, discounting, and pricing in the single-period problem, International Journal of Production Economics 65: 201-216.

Kraus, M. (1982), Highway pricing and capacity choice under uncertain demand, Journal of Urban Economics 12: 122-128.

Massé, P. (1946), Les réserves et la rétulation de l'avenir dans la vie économique, Paris: Hermann, 1946, Vol. 2, Avenir Aléatoire.

McGill, J.I. and van Ryzin, G.J. (1999), Revenue management: research overview and prospects, Transportation Science 33: 233-256.

Meyer, R. A. (1975), Monopoly pricing and capacity choice under uncertainty, American Economic Review 65: 326-337.

Mills, E. A. (1959), Uncertainty and price theory, Quarterly Journal of Economics 73: $116-129$. 
Petruzzi, N.C. and Dada, M. (1999), Pricing and the newsvendor problem: A review with extension, Operations Research 47: 183-194.

Pindyck, R. S. (1982), Adjustment costs, uncertainty, and the behavior of the firm, American Economic Review 72: 415-427.

Polatoglu, L. H. (1991), Optimal order quantity and pricing decisions in single-period inventory systems, International Journal of Production Economics 23: 175-185.

Proost, S. and Van der Loo, S. (2010), Transport infrastructure investment and demand uncertainty, Journal of Intelligent Transport Planning and Operation 14: $129-139$.

Rietveld, P. and Roson, R. (2002), Direction dependent prices in public transport: A good idea? The back haul pricing problem for a monopolistic public transport customer, Transportation 29: 397-417.

Sandmo, A. (1971), The theory of the competitive firm under price uncertainty, American Economic Review 61: 65-73.

Weatherford, L.R. (1997), Using prices more realistically as decision variables in perishable-asset revenue management problems, Journal of Combinatorial Optimization 1: 277-304.

Weitzman, M. I. (1974), Prices vs. quantities, Review of Economic Studies 41: 477491.

Within, T. M. (1955), Inventory control and price theory, Management Science 2: 116-130.

Wong, W., Zhang, A., Hui, YV and Leung, L. (2009), Optimal baggage limit policy: Airline passenger and cargo allocation, Transportation Science 43: 355-369.

Xiao, Y., Fu, X. and Zhang, A. (2013), Demand uncertainty and airport capacity choice, Transportation Research Part B 57: 91-104.

Zabel, E. (1972), Multiperiod monopoly under uncertainty, Journal of Economic Theory 5: 524-536. 
Zhang, A. and Czerny, A. I. (2012), Airports and airlines economics and policy: An interpretive review of recent research, Economics of Transportation 1: 15-34. 


\section{Appendix}

\section{A Varying Uncertainty Types}

Assume that utility is of the linear form discussed in Section 3 and $\alpha=\alpha(\varepsilon)=\varepsilon$. In this example, the uncertainty type is directly determined by the value of the total shock $\varepsilon$ and thus can be positive or negative depending on whether the total shock is positive or, respectively, negative. Assume further that the total shock follows a Bernoulli distribution with $\varepsilon=-\sigma, \sigma$ and $\sigma \leq \min \{\bar{p}, \bar{q}\}$, where each realization occurs with a probability of $1 / 2$. In this scenario, $\sigma$ determines the level of uncertainty as well as the distribution of the total noise shock. Under these conditions, expected profit can be written as $E \pi=\left(\bar{p}+\sigma^{2}\right)\left(\bar{q}-\sigma^{2}\right)$, where the right-hand side increases or decreases in uncertainty if $\bar{q}>\sigma^{2}$ or $\bar{q}<\sigma^{2}$, respectively. The effect of uncertainty on expected profit is maximized for $\sigma=\sqrt{(\bar{q}-\bar{p}) / 2}$; thus, a maximum exists only if $\bar{q} \geq \bar{p}$. Figure 10 displays the difference between profit when the total shock is zero and expected profit as a function of standard deviation $\sigma$ for $\bar{q}=\bar{p}=1$ (dashed line) and $\bar{q}=2, \bar{p}=1$ (solid line) to illustrate these results.

\section{B Proof of Proposition 2}

To apply Cramer's rule, it is useful to denote the determinant of the Hessian of expected profit with respect to size $s$ and quantity $f$ as $\Omega$ with

$$
\Omega \equiv \operatorname{det}\left(\begin{array}{cc}
\frac{\partial^{2} E \pi}{\partial s^{2}} & \frac{\partial^{2} E \pi}{\partial s \partial f} \\
\frac{\partial^{2} E \pi}{\partial s \partial f} & \frac{\partial^{2} E \pi}{\partial f^{2}}
\end{array}\right)
$$

and

$$
\begin{aligned}
\frac{\partial^{2} E \pi}{\partial s^{2}} & =-f^{2} g^{\prime \prime} \\
\frac{\partial^{2} E \pi}{\partial s \partial f} & =\left(\frac{-\Gamma^{\prime}}{2}-\frac{g^{\prime}}{f} \omega(g)\right) f \\
\frac{\partial^{2} E \pi}{\partial f^{2}} & =\left(\left(1-\omega(\Gamma) \frac{(\bar{q}-(1-\alpha) \sigma+s f)}{2 s f}\right)\left(-\Gamma^{\prime}\right)-\frac{g^{\prime}}{f} \omega(g)\right) s,
\end{aligned}
$$




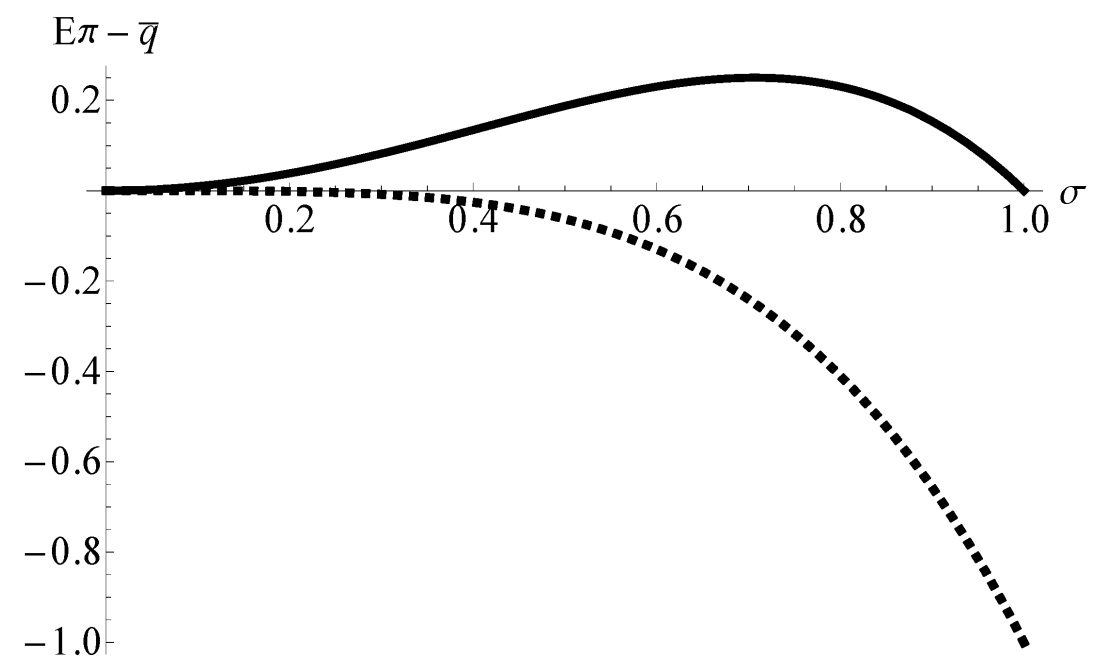

Figure 10: Difference between profit when the total shock is zero and expected profit for $\bar{q}=\bar{p}=1$ (dashed line) and $\bar{q}=2, \bar{p}=1$ (solid line)

where $\Omega>0$ by the second-order conditions and $\partial^{2} E \pi / \partial s \partial f<0$ by Assumption 1 . To derive the effect of uncertainty measured by the standard deviation $\sigma$ on size $s$, Cramer's rule can be applied, which yields

$$
\begin{aligned}
\frac{d s}{d \sigma} & =\frac{1}{\Omega} \operatorname{det}\left(\begin{array}{cc}
-\partial^{2} E \pi / \partial s \partial \sigma & \partial^{2} E \pi / \partial s \partial f \\
-\partial^{2} E \pi / \partial f \partial \sigma & \partial^{2} E \pi / \partial f^{2}
\end{array}\right) \\
& =\frac{1}{\Omega}\left(\frac{\partial^{2} E \pi}{\partial f \partial \sigma} \frac{\partial^{2} E \pi}{\partial s \partial f}-\frac{\partial^{2} E \pi}{\partial s \partial \sigma} \frac{\partial^{2} E \pi}{\partial f^{2}}\right)
\end{aligned}
$$

with

$$
\begin{aligned}
& \frac{\partial^{2} E \pi}{\partial s \partial \sigma}=\frac{\alpha f}{2}, \\
& \frac{\partial^{2} E \pi}{\partial f \partial \sigma}=\frac{1}{2}\left((1-\alpha) \Gamma^{\prime}+\alpha s\right) .
\end{aligned}
$$

The right-hand side of (16) is strictly positive. Furthermore, $\partial^{2} E \pi / \partial f \partial \sigma$ and $(\alpha-\widetilde{\alpha})$ are equal in sign. The second term inside the parentheses on the righthand side of $(15 \mathrm{~b})$ is positive, while the first term inside the parentheses is also positive for $\alpha<\widetilde{\alpha}$. This shows that an increase in uncertainty measured by the standard deviation $\sigma$ increases the optimal size $s$ when the quantity $q$ is sufficiently uncertain. 
Analogously, the effect of an increase in uncertainty on the optimal quantity $f$ can be derived as:

$$
\frac{d f}{d \sigma}=\frac{1}{\Omega}\left(\frac{\partial^{2} E \pi}{\partial s \partial \sigma} \frac{\partial^{2} E \pi}{\partial s \partial f}-\frac{\partial^{2} E \pi}{\partial f \partial \sigma} \frac{\partial^{2} E \pi}{\partial s^{2}}\right) .
$$

The first term inside the parentheses is negative, while the second term inside the parentheses is also negative for $\alpha<\widetilde{\alpha}$. This shows that an increase in uncertainty measured by the standard deviation $\sigma$ reduces the optimal quantity $f$ when the quantity $q$ is sufficiently uncertain, which completes the proof.

\section{Welfare Example: "Prices vs. Quantities"}

Assume that passenger demand is implicitly determined by the equilibrium condition $B^{\prime}=p$, and that there are external costs of consumption, denoted $C$ with $C=C(q)$. Social welfare, denoted $W$, can then be written as $W=B-C$. The welfare-optimal quantity, denoted $q^{*}$, is determined by the first-order condition $B^{\prime}=C^{\prime}$, where the marginal benefits of consumption are equal to the marginal external costs of consumption. Under perfect information, the first-best result can be achieved by using quantity-related instruments where the policy maker imposes an upper limit on consumption $q$ given by $q^{*}$ such that $q \leq q^{*}$ and by ensuring that individuals with high benefits from consumption consume first (assume that "consumption rights" are auctioned off), or by using price-related instruments where the policy maker charges a price $p^{*}=C^{\prime}\left(q^{*}\right)$ to customers.

Assume that the benefit function is of a quadratic form described in Section 3. Assume further that the policy maker has perfect information about the demand slope, $b$, but is imperfectly informed about the choke price, $a$, and that the external cost is of the quadratic form $C=v q^{2} / 2$ with $v>0$, where $v$ is known to the policy maker. Figure 11 illustrates this situation. It shows a high and a low inverse demand function $\left(B^{\prime}\right)^{\prime}$ and $\left(B^{\prime}\right)^{\prime \prime}$ with $\left(B^{\prime}\right)^{\prime}<\left(B^{\prime}\right)^{\prime \prime}$, while the vertical difference between the two inverse demands is independent of consumption $q$. The figure also displays marginal external costs, $v q$, and the corresponding welfare-optimal price/quantity pairs $\left(p^{*}, q^{*}\right)^{\prime}$ and $\left(p^{*}, q^{*}\right)^{\prime \prime}$. In the scenario displayed in Figure 11, the inverse 


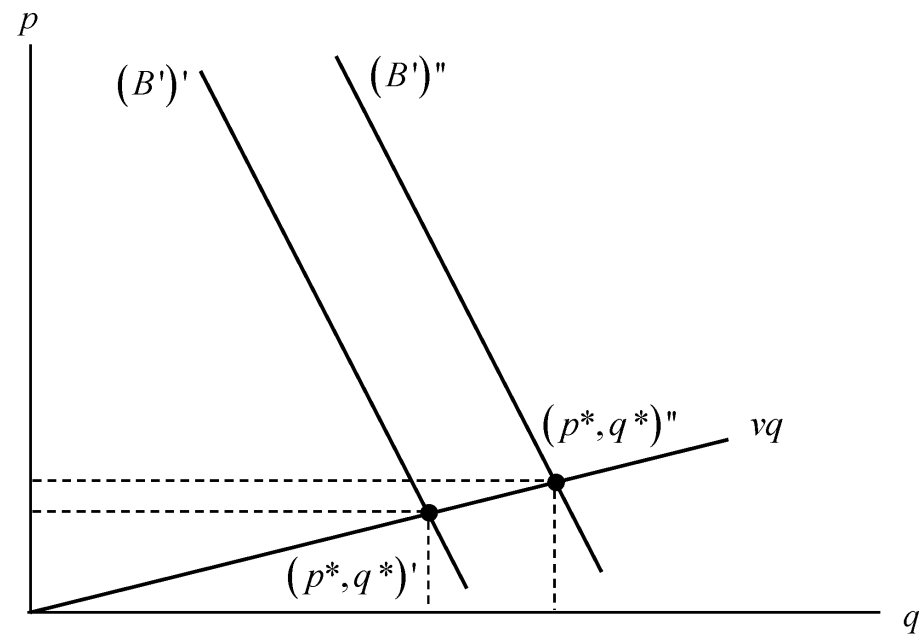

Figure 11: Demand is steep relative to marginal external congestion costs, which means that prices should be preferred over quantities

demands are steep relative to the marginal external costs function, i.e., $v<b$. In this instance, Weitzman's (1974) result on the topic can be used, which showed that under these conditions the risk-neutral policy maker is better off with price rather than quantity related instruments.

To see how this relates to our analysis on the stochasticity of optimized values, consider the horizontal and the vertical dashed lines, which indicate the optimized prices and quantities, respectively. Observe that the variation of optimized prices is small relative to the variation of optimized quantities. In this sense, the optimized prices are easier to predict than the optimized quantities and it therefore seems intuitive that price instruments should be preferred to quantity-related instruments from the social viewpoint. However, note that Weitzman's results can only be applied to situations where slopes are deterministic and constant in consumption, which seems a strong set of assumptions. The following example is used to illustrate how the explicit consideration of uncertainty types can be used to analyze situations with linear but stochastic slopes.

Assume that benefits are quadratic with deterministic choke price and stochastic demand slope $b=(a-(\bar{p}+\alpha \varepsilon)) /(\bar{q}+(1-\alpha) \varepsilon)$, and marginal externality costs 
are linear with stochastic slope $v=(\bar{p}+\alpha \varepsilon) /(\bar{q}+(1-\alpha) \varepsilon)$, which ensures that for welfare-optimal prices and quantities it holds that $p^{*}=(\bar{p}+\alpha \varepsilon)$ and $q^{*}=$ $\bar{q}+(1-\alpha) \varepsilon$. Thus, if $\alpha=0$, the welfare-optimal price is deterministic, while the welfare-optimal quantity is stochastic. In this scenario, the first-best result can thus been reached for all values of $\varepsilon$ by the choice of prices with $p=\bar{p}$. For an intuition, consider parameters $b$ and $v$ for $\alpha=0$, which yields $b=(a-\bar{p}) /(\bar{q}+\varepsilon)$ and $v=\bar{p} /(\bar{q}+\varepsilon)$. Observe that both $b$ and $v$ are decreasing in the total shock $\varepsilon$. This means that demand and external costs are negatively correlated in this case, which is a condition that favors the use of pricing mechanism as described Weitzman (1974). On the other hand, if $\alpha=1$, the welfare-optimal price is stochastic, while the welfare-optimal quantity is deterministic, which means that the first-best result can be reached for all values of $\varepsilon$ by the choice of quantities with $q=\bar{q}$. For an intuition, consider parameters $b$ and $v$ for $\alpha=1$, which yields $b=(a-(\bar{p}+\varepsilon)) / \bar{q}$ and $v=(\bar{p}+\varepsilon) / \bar{q}$. Observe that both $b$ is decreasing in the total shock $\varepsilon$, while $v$ is increasing in the shock $\varepsilon$. This means that demand and external costs are positively correlated in this case, which is a condition that favors the use of quantity mechanism as described Weitzman (1974). This shows that Weitzman's result on the effect of correlations between demand and cost functions carries over to the case with stochastic slopes. 